\title{
RETRACTION: Antigen-induced tolerance by intrathymic modulation of self-recognizing inhibitory receptors
}

Yoshihiro Hayakawa, Stuart P Berzins, Nadine Y Crowe, Dale I Godfrey \& Mark J Smyth

Nat. Immunol. 5, 590-596 (2004)

We are retracting this paper because it contains a number of errors, including duplications of some flow cytometry plots in Figures $\mathbf{1}, \mathbf{3 a}$ and $\mathbf{4}$ and Supplementary Figure 1 online and incorrect reporting of ' $n$ ' and s.e.m. values that in some cases weakens the statistical significance. Although original data exist for each figure that largely support the conclusions drawn, we believe that the number of figures affected means that the appro할 priate response is to retract this paper. 